\title{
ENTEROBACTERIAS COMO AGENTES ETIOLOGICOS DE LA DIARREA EN LA COMUNIDAD
}

\author{
Carlos Agudelo*, Hilda Viveros *, Elizabeth Castañeda**.
}

\begin{abstract}
Con el fin de identificar, a nivel de la comunidad, los enteropatógenos bacterianos que producen diarrea en niños menores de 10 años, tomamos muestras de heces de 204 pacientes con cuadro diarreico de 1 a 7 días de evolución y de un grupo control, sin diarrea, conformado por 48 niños. Evaluamos también la recuperación de los microorganismos empleando o no medio de Cary-Blair como medio de transporte e identificamos las categorías de Escherichia coli aisladas empleando pruebas biológicase inmunológicas. Se destacaron como agentes etiológicos de la diarrea E. coli $(58,8 \%)$, E. coli enterotoxigénica, productora de toxina labil (ECET-TL) $(5,1 \%)$, Salmonella enteritidis $(6,9 \%)$ y Shigella sonnei $(5,9 \%)$. Cuando no se usó el medio de transporte de CaryBlair la sensibilidad en la recuperación de los microorganismos fue del 37\%. Se destaca la importancia de determinar la frecuencia real de las categorías de $E$. coli en nuestro medio.
\end{abstract}

\section{INTRODUCCION}

La enfermedad diarreica aguda de origen bacteriano, constituye un problema de salud pública que tiene una resonada importancia por su impacto en la morbilidad y mortalidad infantil, asi como por la severidad de los cuadros clínicos (1).

En varios países se han realizado estudios para identificar las enterobacterias que juegan un papel determinante en el síndrome diarreico, pero la gran mayoría de ellos analizan población hospitalaria cuyos cuadros son de mayor intensidad y gravedad pero que representan solo una parte de quienes padecen la enfermedad en la comunidad (1-5).

Actualmente, uno de los aspectos mas sobresalientes de los estudios etiológicos es la clasificación de las cepas de Escherichia coli en categorías basadas en el mecanismo de producción de diarreas. Estas categorías son : $E$. coli enterotoxigénica (ECET), enteroinvasiva
(ECEI), enteropatógena (ECEP), enterohemorrágica (ECEH) y enteroadherente (ECEA (6). Para realizar esta clasificación se cuenta en el laboratorio con un conjunto de pruebas biológicas, consideradas como pruebas de referencia $(7,8)$, serológicas (7), en cultivos celulares $(9,10)$, inmunológicas $(7,11-14)$ y de biología molecular empleando sondas de DNA (15-19).

En Colombia se han publicado cinco estudios etiológicos en los últimos diez años (20$24)$. En dos se identificó $E$. coli enteropatógena (ECEP) por medio de serotipificación $(22,24)$; en otro se identificó $E$. coli sin diferenciar sus cepas; otro utilizó serotipificación y una prueba inmunológica (Biken) para identificar $E$. coli productora de toxina termolabil (ECET) (24) y otro identificó las cepas de E. coli por medio de hibridización con DNA (23).

En el presente estudio se propuso identificar a nivel de comunidad las enterobacterias que producen diarrea para establecer su frecuencia y algunos de los factores epidemiológicos;

\footnotetext{
* Departamento de Microbiología,Facultad de Medicina, Universidad Nacional de Colombia, Bogotá.

** Grupo de Microbiología, Instituto Nacional de Salud,Santafé de Bogotá.
} 
evaluar diferentes métodos de transporte de las muestras de materia fecal y su procesamiento en el laboratorio e identificar las cepas de E. coli y clasificarlas en las categorias establecidas.

\section{MATERIALES Y METODOS}

Población. Se tomaron muestras de heces de 204 niños menores de diez años, de una comunidad de escasos recursos de Bogotá, quienes acudieron a consulta ambulatoria por presentar diarrea, definida como la presencia de tres o más deposiciones líquidas o semilíquidas en 24 horas, de 1 a 7 días de duración, entre junio de 1987 y junio de 1988. Los pacientes no habían recibido antibióticos en los dos meses anteriores a la toma de la muestra y su ingreso al estudio fue autorizado por los familiares.

Igualmente, se tomaron muestras de materia fecal de 48 niños de la misma edad, y residentes en la misma comunidad que consultaron por razones distintas a la diarrea, los cuales conformaron el grupo control.

Variables. De cada uno de los sujetos a estudio se obtuvo información sobre sexo, edad, estado nutricional, síntomas, hábitos higiénicos, vivienda, fuente de agua, eliminación de excretas y posesión de animales.

Toma, transporte y procesamiento de las muestras e identificación de los enteropatógenos. El procedimiento empleado para el transporte de todas las muestras fue el uso del medio de transporte de Cary y Blair (BBL) en el cual se introdujeron dos escobillomes tratados con carbón activado (Merck) e impregnados con las muestras de heces. El procesamiento de las muestras transportadas se realizó en las tres horas siguientes a la toma de la muestra.

En una submuestra de 48 casos del grupo de pacientes una porción de las heces se depositó en un recipiente y se transportó al laboratorio en condiciones refrigeradas, en donde se procesaron dentro de un lapso de dos horas.

También se obtuvieron de todos los pacientes muestras por frotis rectal las cuales se sembraron de inmediato en el medio de Campy -bap (Oxoid) y como medio de enriquecimiento se empleó Campy-tio (Oxoid). Los medios inoculados fueron transportados al laboratorio en una atmósfera de $\mathrm{CO}_{2}$ al 3-5\% (jarra con vela).

El procesamiento de las muestras incluyó las siembras en agar Mac Conkey (Oxoid), agar Hecktoen (Merck), agar xilosa-lisinadesoxicolato (BBL) y en caldo de selenito (Merck) para enriquecimiento, el caldo se incubó a $37^{\circ} \mathrm{C}$ durante $18-24$ h y a partir de el se subcultivó en los agares descritos anteriormente. Se realizó tambien enriquecimiento a $4^{\circ} \mathrm{C}$ en solución salina buffer pH 7,6 durante 14 a 21 días para la recuperación de Yersinia sp. a partir de la cual, posteriormente se sembró en los medios adecuados. Después de la incubación de los medios sólidos a $37^{\circ} \mathrm{C}$ durante $24 \mathrm{~h}$, se realizó una identificación presuntiva de los enteropatógenos con base en las reacciones en agar triple azúcar hierro (TSI) (Merck) y agar lisina hierro (LIA) (Merck). La identificación final se realizó empleando las pruebas bioquímicas estandarizadas para tal fin (25).

Las muestras tomadas por frotis rectal se incubaron en el medio de enriquecimiento a $4^{\circ} \mathrm{C}$ durante 8-18 h, luego se subcultivaron en Campybap o en medio libre de sangre (Oxoid), incubandose $\mathrm{a} 42^{\circ} \mathrm{C}$ durante $24-72 \mathrm{~h}$, en atmósfera microaerofílica $\left(10 \% \mathrm{CO}_{2}, 5 \% \mathrm{O}_{2}\right.$ y $\left.85 \% \mathrm{~N}_{2}\right)$. La identificación final se realizó con base en las reacciones bioquímicas descritas para el género Campylobacter (25).

Serología. Las cepas de Salmonella fueron serotipificadas empleando la prueba de aglutinación con antisueros específicos (Behring), para las cepas de Shigella se emplearon antisueros polivalentes para los grupos A,B,C y D (Behring). Los serotipos de E. coli fueron determinados en el total de las cepas aisladas empleando los antisueros polivalentes Bacto $E$. coli OK poly A, B, C, D, y E (DIFCO). La serotipificación fue realizada con una colonia de cada muestra.

Caracterización de las cepas de $E$ coli. Se realizaron las pruebas biológicas descritas en el Manual de laboratorio de infecciones entéricas agudas de la Organización Mundial de la Salud (7). Las pruebas empleadas fueron: 
1. Asa ligada de conejo para determinar las cepas productoras de toxina termolábil (ECET-TL). La prueba se realizó en 116 cepas. Las cepas positivas fueron confirmadas con la prueba de coaglutinación (ETECLT, Pharmacia).

2. Prueba de ratón lactante para determinar las cepas productoras de toxina termoestable (ECET-TE). En esta prueba se empleó como contro negativo la cepa $E$. coli-9 de la Corporación para Investigaciones Biológicas (CIB) de Medellín. Los ensayos de estandarización con esta cepa determinaron el nivel de positividad en un valor mayor o igual a $0,110(8,27)$. La prueba se realizó con 88 cepas.

3. La prueba de Sereny en ojo de conejo para determinar las cepas invasivas $(7,30)$. En esta prueba se empleó como control positivo una cepa de Shigella y se probaron 101 cepas de E. coli .

Análisis estadístico. En el análisis de todas las variables y sus relaciones se utilizaron la prueba de chi cuadrado y de z. De acuerdo con la naturaleza de las variables, el número de las mismas y su frecuencia, se utilizaron las pruebas de Fisher, Cramer, lambda, Phi y el coeficiente de contingencia (31).

\section{RESULTADOS}

Frecuencia de aislamientos de Enterobacterias. E coli presentó la mayor frecuencia de aislamiento en el grupo de pacientes y en el grupo control $(58,8 \%$ y $37,5 \%)$, seguida, en el grupo de estudio, por la cepa toxigénica productora de toxina termoestable (ECET-TE) $(35,1 \%)$ y ECEP $(29,2 \%)$. Las dos últimas clases de E. coli presentaron también una elevada frecuencia en el grupo control $(27,3 \%$ y $38,8 \%)$ no siendo la diferencia entre los dos grupos significativa. Por el contrario, las cepas toxigénicas productoras de toxina termolabil (ECET-TL) y ECEI se aislaron solamente en el grupo de estudio $(5,1 \%$, 1\%) (Tabla 1). La prueba de coaglutinación fue positiva en todas las cepas de ECET-TL determinadas por la prueba biológica.

En ninguna de las cepas de $E$. coli estudiadas se pudo determinar la presencia de ambas toxinas (TL y TE) ni se pudo determinar la presencia de dos categorias de $E$. coli en el mismo paciente.

Las cepas de ECEP se presentaron en ambos grupos, con una mayor frecuencia en el grupo control. En las 35 cepas del grupo de estudio caracterizadas como ECEP los serotipos identificados fueron $018 \mathrm{ab}, \mathrm{O} 18 \mathrm{ac}, \mathrm{O} 26: \mathrm{K} 60$, O111:K58, O119:K69, O128:K67, O125:K70 y O127a:K63; en este último serogrupo se clasificaron 16 cepas $(45,7 \%)$. En el grupo control los serotipos de las siete cepas fueron: una 0125:K70, dos 0111:K58 y cuatro 0127a:K63.

En la Tabla 1 se percibe que de las 120 muestras positivas para E. coli en el grupo en estudio solo 67 (56\%) pudieron ser clasificadas en una de las categorías estudiadas; en el grupo control fueron clasificadas 10 de 18 $(55,5 \%)$.

Se logró aislar Campylobacter, identificado como $C$. jejuni en $10,3 \%$ del grupo de estudio y en $6,3 \%$ del grupo control (Tabla 1). Los aislamientos de $S$. enteritidis y $S$. sonnei sólo se obtuvieron en el grupo de estudio y constituyeron el 6,9 y $5,9 \%$ de los aislamientos (Tabla 1 ). No se aislóYersinia sp. en ninguno de los dos grupos.

Se presentó con alguna frecuencia asociación entre las enterobacterias. Con respecto a las 204 muestras del grupo de estudio, cinco de ellas $(2,5 \%)$ presentaron la asociación $C$. jejuni

\begin{tabular}{|c|c|c|c|c|}
\hline \multicolumn{5}{|c|}{$\begin{array}{c}\text { TABLA } 1 \\
\text { FRECUENCIA DE ENTEROPATÓGENOS SEGUN GRUPO. }\end{array}$} \\
\hline MICROORGANISMO & \multicolumn{2}{|c|}{$\begin{array}{l}\text { GRUPO DE ESTUDIO } \\
\text { POSITIVOS }\end{array}$} & \multicolumn{2}{|c|}{$\begin{array}{l}\text { GRUPO CONTROL } \\
\text { POSITIVOS }\end{array}$} \\
\hline E. coli & $120 / 204$ & 58,8 & $18 / 48$ & 37,5 \\
\hline ECEP & $35 / 120$ & 29,2 & $7 / 18$ & 38,8 \\
\hline ECET-TE & $26 / 74$ & 35,1 & $3 / 14$ & 27,3 \\
\hline ECET-TL & $5 / 98$ & 5,1 & $0 / 18$ & 0,0 \\
\hline ECEI & $1 / 98$ & 1,0 & $0 / 3$ & 0,0 \\
\hline C. jejuni & $21 / 204$ & 10,3 & $3 / 48$ & 6,3 \\
\hline S. enteritidis & $14 / 204$ & 6,9 & $0 / 48$ & 0,0 \\
\hline S. sonnei & $12 / 204$ & 5,9 & $0 / 48$ & 0,0 \\
\hline
\end{tabular}


y E. coli y en otras cinco se encontraron estas dos bacterias junto con $S$. sonnei. En dos muestras se presentó la asociación $S$. enteritidis, $E$. coli y en igual número estas dos bacterias junto con C. jejuni.

Las diferencias entre el grupo de estudio y de control fueron significativas en los siguientes casos: $S$. enteritidis $(\mathrm{z}=3.9 \mathrm{p}<0.01)$. $S$. sonnei $(\mathrm{z}=3.6 \mathrm{p}<0.01)$. E. coli sin diferenciar $(\mathrm{z}=2.5$ $\mathrm{p}<0.02)$ y ECET-TL $(\mathrm{t}=2.3 \mathrm{p}<0.03)$.

Comparación de métodos. Los diferentes métodos de procesamiento de las muestras se aplicaron para los aislamientos de $E$. coli y Salmonella $s p$ en 48 muestras. En el caso de $E$. coli se empleó el transporte en Cary-Blair como método diagnóstico (prueba estandard) y el transporte en el recipiente como método de prueba ( Tabla 2 ). De estos resultados se desprende que el procedimiento de transportar la muestra sin emplear el medio de transporte tiene una sensibilidad de $37 \%$, una especificidad de $71 \%$, un valor predictivo positivo de $62,5 \%$, un valor predictivo negativo de $46,8 \%$ y una eficacia de $52 \%$. En el caso del aislamiento de $S$. enteritidis sólo el empleo del medio de transporte permitió la recuperación de la bacteria.

\begin{tabular}{|l|l|}
\hline \multicolumn{4}{|c|}{ TABLA 2 } \\
\multicolumn{3}{|c|}{ COMPARACIÓN DE DOS MÉTODOS PARA EL TRANSPORTE } \\
DE LAS MUESTRAS DE HECES.
\end{tabular}

Variables. Edad: relacionando los aislamientos por grupos de edad, los porcentajes de positividad se indican en la Figura 1. Destaca la mayor positividad de $E$. coli y de $C$. jejuni en los menores de 1 año y de $S$. enteritidis y de $S$. sonnei en los niños de 1 a 2 años.
Signos y síntomas: en el grupo de estudio, el $85,1 \%$ presentó diarrea durante 3 ó más días y el $34,2 \%$ durante 8 o más días. Sólo un caso del grupo de estudio presentó deshidratación moderada, mientras que $181(88,7 \%)$ pacientes presentaron deshidratación leve; el 10,8\% restante no presentó signos de deshidratación.

Como se observa en la Figura 2, el orden de frecuencia de los signos y síntomas fue, de mayor a menor: pérdida de apetito, dolor abdominal, fiebre, heces fétidas, vómito y moco en las heces.

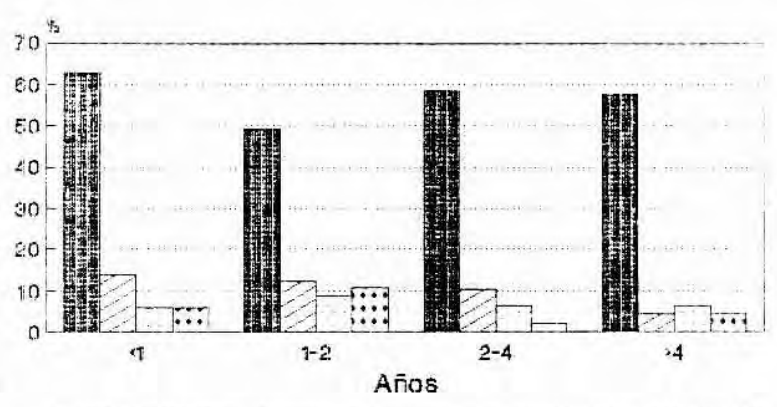

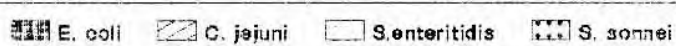

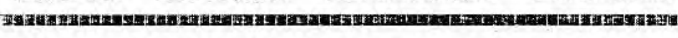

Figura 1. Porcentajes de positividad por edades

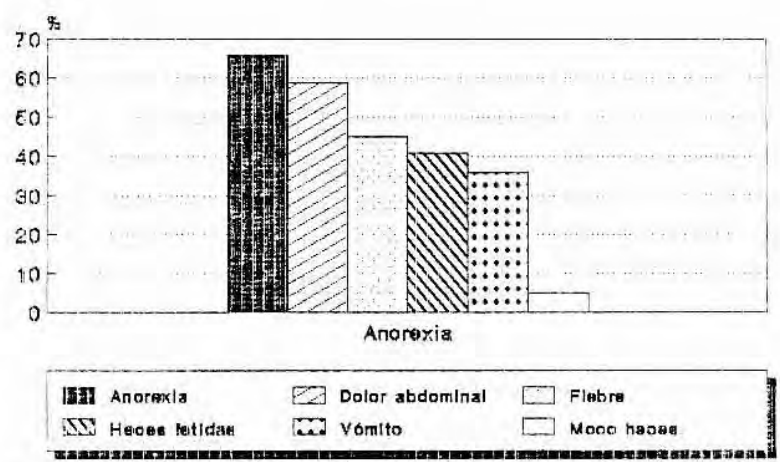

Figura 2. Síntomas principales en los pecientes con diarrea

Relaciones entre las variables. En la Tabla 3 se indican los factores con los cuales las enterobacterias detectadas presentaron una mayor frecuencia, por lo que pueden considerarse como factores de riesgo. 


\begin{tabular}{|llcc|}
\hline \multicolumn{4}{c|}{ TABLA 3 } \\
FACTORES CON LOS CUALES LAS ENTEROBACTERIAS \\
PRESENTARON MAYOR FRECUENCIA. \\
PRUEBA DE CHI-CUADRADO. \\
\hline Bacteria & Factor & $X^{2}$ & P \\
E. coli & Consumo de leche hervida & 10 & 0.006 \\
ECEI & Intervalos de aseo largos(1) & 8 & 0.046 \\
& Heces color ocre & 8 & 0.046 \\
ECET-TL & Dolor abdominal & 9 & 0.011 \\
Campylobacter & Agua no potable & 6.6 & 0.037 \\
& Sin alcantarillado & 8.1 & 0.043 \\
& Diarrea de 4 dias o más & 19.7 & 0.006 \\
Salmonella & Heces con olor fétido & 10.9 & 0.028 \\
& Intervalos de aseo largos(2) & 16.8 & 0 \\
Shigella & Agua no potable & 15 & 0 \\
& Intervalos de aseo largos(2) & 21.9 & 0.001 \\
& Posesión de animales(3) & 18.2 & 0.006 \\
& Heces con olor fétido & 12.3 & 0.015 \\
& Heces con moco abundante & 6.8 & 0.033 \\
\hline (1) Del niño & & & \\
(2) De la vivienda & & & \\
(3) Diferentes de perros, gatos, cerdos y gallinas & & \\
\hline
\end{tabular}

\section{DISCUSION}

A nivel de la comunidad estudiada, se destacan de manera clara como agentes etiológicos de la diarrea E. coli, ECET-TL, S. sonnei y $S$. enteritidis. Estos hallazgos no disminuyen la importancia de otras enterobacterias como causantes de diarrea sino que señalan que las condiciones de la comunidad estudiada no son lo suficientemente específicas en tanto los factores de riesgo operan de manera amplia.

Con fines de comparación se indican a continuación las enterobacterias detectadas en otros estudios colombianos (Tabla 4). En cuatro de estos estudios los pacientes fueron niños consultantes a servicios hospitalarios (20$22,24)$, aunque uno de ellos utilizó también un grupo que fue atendido en un centro de salud ambulatorio (24). Uno de los estudios fue realizado en una comunidad de escasos recursos de Bogotá (23).

Con respecto a los patrones encontrados en población hospitalaria las diferencias se refieren tanto al tipo de agentes, como a sus frecuencias. Las diferencias en las frecuencias relativas de las enterobacterias destacan, y son consecuencia de las diversas condiciones ambientales y climáticas de las épocas del año, del tipo de población analizada, de los procedimientos de toma y transporte de las muestras, y de las técnicas de aislamiento e identificación utilizadas en el laboratorio. Esto último puede tener considerable importancia cuando se trata de bacterias como especies de Salmonella, Shigella, o de Campylobacter el cual fue aislado con una frecuencia importante en los dos grupos que estudiamos y el cual requiere para su transporte y crecimiento una atmósfera microaerofílica. Es así como la comparación de los métodos de toma de las muestras, de transporte y de procesamiento, en nuestro estudio, demostró que sólo aquellos de tipo específico y que proveían las condiciones óptimas para el transporte de las muestras fueron útiles para la recuperación de los agentes etiológicos.

Con respecto al estudio realizado con una comunidad de Bogotá, nuestro estudio obtuvo frecuencias más elevadas en todas las enterobacterias aisladas con excepción de Yersinia sp. la cual no fue recuperada en nuestro grupo en estudio. En estas diferencias deben haber pesado las condiciones económicas, sociales y ambientales de las comunidades estudiadas y los diferentes métodos de diagnóstico.

La clasificación de $E$. coli en sus diversas categorías destacó la importancia de ECEP y ECET productora de ambas toxinas. La ECEP se presentó con frecuencia similar a la informada en algunos de los estudios colombianos publicados $(20,22,24)$. Con relación a la ECET productora de toxina labil nuestros datos, empleando las pruebas biológica e inmunológica (coaglutinación), son similares a los obtenidos

\begin{tabular}{|llrrrrr|}
\hline \multicolumn{7}{c|}{ TABLA 4 } \\
FRECUENCIAS (\%) \\
ESTUDIOS ENTEROBACTERIAS SEGÚN \\
Agentes & 1 & 2 & 3 & 4 & 5 \\
\hline E. coli & n.d. & 22,8 & n.d. & 5,4 & 90.0 \\
ECEP & 28,0 & n.d. & 14,4 & 0,7 & 25,0 \\
ECET-TL & n.d. & n.d & n.d. & 3,8 & 0.0. \\
ECET-TE & n.d. & n.d. & n.d. & 0,9 & n.d. \\
ECEI & n.d. & n.d. & n.d. & 0,0 & 0,0 \\
Campylobacter sp. & 0,0 & n.d. & 1,6 & 0,3 & n.d. \\
Salmonella sp. & 0,0 & n.d. & 0,0 & 0,9 & 1,0 \\
Shigella sp. & 0,0 & n.d. & 7,2 & 4,4 & 1,0 \\
Yersinia sp. & 0,0 & n.d. & 0,0 & 0,2 & n.d. \\
& & & & & & \\
\hline No. de casos & 25 & 144 & 180 & 588 & 100 \\
Referencia & $(20)$ & (21) & $(22)$ & $(23)$ & $(24)$ \\
& & & & & & \\
\hline n.d.: no disponible & & & & & \\
\hline
\end{tabular}


en el estudio en la comunidad, en el cual se emplearon sondas de DNA en pruebas de hibridización (23); vale la pena destacar la sensibilidad y especificidad de la prueba inmunológica frente a la prueba biológica, lo cual la coloca como una prueba ideal para el estudio de la ECET-TL por la sencillez de su realización la cual puede ser establecida en un laboratorio de complejidad intermedia. Por el contrario existen diferencias en las frecuencias de ECET productoras de toxina estable $35,1 \%$ en nuestro estudio y $0,9 \%$ en el estudio mencionado anteriormente, las cuales se pueden atribuir a las diferencias en metodologias, los datos de clasificación de las diferentes categorías de E.coli también señalan la importancia de seguir investigando su frecuencia real en diferentes poblaciones de nuestro medio, empleando para ello las diferentes técnicas con las que cuenta el laboratorio actual. Vale la pena destacar el hallazgo de $E$. coli enteropatógena y ECET-TL en el grupo control, para ser considerado en futuros trabajos.

Por edades, los porcentajes de positividad indican que los agentes patógenos tienden a concentrarse en el grupo menor de 4 años. En este aspecto, sin embargo, no es posible establecer comparaciones ya que los estudios nacionales no señalan la correspondiente información.

Los síntomas más importantes corresponden con los cuadros descritos en otros estudios, tanto a nivel de comunidad como hospitalarios, lo cual resta valor a la sintomatología como instrumento para diferenciar la severidad de las afecciones, cuando se trata de comparar grupos. Se logró establecer la relación de ciertos agentes y algunos signos o síntomas; los posibles patrones de relaciones, sin embargo, están lejos de la amplitud y la especificidad de los modelos teóricos. Los factores de riesgo detectados son similares o corresponden al mismo grupo de factores que los encontrados en otro estudio de comunidad (23): hábitos higiénicos, posesión de animales, fuente de agua y eliminación de excretas. Pero se logró una mayor especificidad en cuanto a los posibles agentes bacterianos más directamente relacionados con dichos factores de riesgo. Cabe destacar que la $E$. coli fue más frecuente cuando se hervía la leche, lo que sugiere manipulación y almacenamiento inadecuados.
Finalmente señalamos la importancia de la realización de este tipo de estudios para poder obtener los datos adecuados para implementar las medidas de salud requeridas.

\section{SUMMARY}

In order to determine the bacterial agents causing diarrhea in a community, we studied 204 stool samples from children with diarrhea less than ten years old and 48 controls. We also evaluated the utility of the Cary-Blair transport medium and determined the categories of the strains of E. coli isolated using biological and immunological techniques. We found $E$. coli, enterotoxigenic E.coli heat labile toxin producer (ETEC-LT), Salmonella enteritidis and Shigella sonnei as the principal etiological agents with frequencies of $58.8 \%, 5.1 \%, 6.9 \%$ and $5.9 \%$ respectively. Without the transport medium the sensitivity of the recovery was $37 \%$. The relevance of studies to determine the real frequencies of the categories of E. coli is established.

\section{AGRADECIMIENTOS}

Agradecemos la ayuda técnica de las bacteriólogas Mercedes de Guevara, María Consuelo Garzón y Nélida Muñoz en el procesamiento de las muestras. Al Doctor Jaime Robledo de la Corporación de Investigaciones Biológicas de Medellín, por sus valiosos comentarios al revisar el manuscrito.

\section{BIBLIOGRAFIA}

1. Guerrant RL,Moore RA,Kirschfeld PM.Sande MA.Role of toxigenic and invasive bacteria in acute diarrhea of childhood. N Engl J Med 1975; 293: 567.

2. Mc Lean M, Brennan R, Hughen JM, Korzeniowski OM, et al.Etiología de la diarrea infantil y terapia de rehidratación oral en el nordeste de Brasil. Bol Of Sanit Panam 1982; 92: 405.

3. Kourany M, Vásquez MA. Housing and certain socioenvironmental factors and prevalence of enteropathogenic bacteria among infants with diarrheal disease in Panama. Am JTrop Med Hyg 1969; 18:936. 
4. Aislamiento de enterobacterias patógenas en niños con procesos diarreicos agudos,Argentina, 1971-1978. Boletin Epidemológico. Organización Panamericana de la Salud 1982; $3: 7$.

5. Riverón RC, Gutiérrez JA. Enfermedades diarreicas agudas en América Latina, 1970-1979. La situación en Cuba. Bol Of Sanit Panam 1982; 92: 508.

6. Schlager TA, Guerrant RL. Seven possible mechanisms for Escherichia coli diarrhea. Infect Dis Clinics of North America $1988 ; 2: 607$.

7. Organización Panamericana de la Salud. Organización Mundial de la Salud. Manual de investigaciones de laboratorio de infecciones entéricas agudas. Washington D.C., 1983.

8. Dean AG, Ching YC, Wiliams RG, et al. Test for Escherichia coli enterotoxin using infant mice: application in a study of diarrhea in children in Honolulu. J Infect Dis $1972 ; 125: 407$.

9. Guerrant RL,Brunton LL, Schaitman TL, et al. Cyclic adenosine monophosphate and alteration of Chinese hamster ovary cell morphology. Infect Immun 1974; 10: 320.Sack DA,

10. Sack RB. Test for enterotoxigenic Escherichia coli using Y1 adrenal cells in miniculture. Infect Immun 1975;11:334.

11. Sack DA, Huda S, Neogi PKB, Daniel RR,Spira WM. Microtiter ganglioside Enzyme-Linked Immunosorbent Assay for Vibrio and Escherichia coli heat labile enterotoxins and antitoxin. J Clin Microbiol 1980; 11: 35.

12. Honda T, Glass RI, Akhtar Q, et al. A simple assay to detect Escherichia coli producing heat labile enterotoxin: results of a field study of the Biken test in Bangladesh. Lancet 1981; 2: 609 .

13. Honda T, Taga S, Takeda Y, et al. Modified Elek test for detection of heat-labile enterotoxin of enterotoxigenic Escherichia coli. J Clin Microbiol 1981; 13: 1.

14. Vadivelu J, Drasar BS, Cox NP, et al. Membrane filter assay for detection of enterotoxigenic Escherichia coli in epidemiologic studies. Lancet 1986; i:1007.

15. Moseley SL, Huq I, Alim ARMA, et al. Detection of enterotoxigenic Escherichia coli by DNA colony hybridization. J Infect Dis 1980; 142: 892.

16. Moseley SL, Echeverria P, Seriwatana J.et al. Identification of enterotoxigenic Escherichia coli by colony hybridization using three enterotoxin gene probes. J Infect Dis 1982; 145: 863.

17. Echeverria P, Seriwatana J, Taylor DN, et al. Identification by DNA hybridization of enterotoxigenic Escherichia coli in homes of children with diarrhoea.Lancet 1984;1: 63 .
18. Sethabutr $\mathbf{O}$, Hanchalay $\mathbf{S}$, Echeverria $\mathbf{P}$, et al. A nonradioactive DNA probe to identify Shigella and enteroinvasive Escherichia coli in stools of children with diarrhea. Lancet 1985; 2: 1095.

19. Taylor DN, Echeverria P, Pitarangsi C, et al. Application of DNA hybridization techniques in the assessment of diarrheal disease among refugees in Thailand. Am J Epidemiol 1988; 127: 179

20. Trujillo H, Jaramillo C, Restrepo M, et al. Rotavirus y otros enteropatógenos en la etiología de la diarrea aguda en Medellín. Colombia, 1982. Bol Of Sanit Panam 1985; 98:251.

21. Hernández A, Jaramillo TC, Ramírez SR, et al. Tratamiento de diarrea aguda en niños. Bol Of Sanit Panam 1987; 102:606.

22. Leal F, Franco G, Sandoval CI, et al. Agentes etiológicos de la diarrea aguda en Bogotá. Pediatría 1985;21:7

23. Mora JO, Suescun J, Juliao O, et al . Estudio longitudinal sobre la etiología y epidemiología de la enfermedad diarreica aguda en los niños de una comunidad urbana pobre de Bogotá, Colombia. Serie Publicaciones Científicas No. 15. Instituto Nacional de Salud. Bogotá, 1988.

24. GómezCL,RobledoJ,Mejía GI,etal. E.coli enterotoxigénico productor de la toxina termolábil como causante de enfermedad diarreica aguda en Medellín. Laboratorio Actual 1989; 17:15.

25. Instituto Nacional de Salud. Microbiología Médica. Manual de procedimientos. Publicaciones científicas No. 14. Bogotá, 1988.

26. Sack RB, Hirschhorn N, Brownlee I, et al. Enterotoxigenic Escherichia coli associated diarrheal disease in apache children. N Engl J Med 1975;292:1041.

27. Shore EG, Dean AG, Holik KJ, et al. Enterotoxin- producing Escherichia coli and diarrheal disease in adult travelers.J Infect Dis 1974; 129: 577.

28. Bradley RS, Gorbach SL, Banwell JG, et al. Enterotoxigenic Escherichia coli isolated from patiens with severe choleralike disease. J Infect Dis 1971; 123:378.

29. Dupont HL, Formal SB, Hornick RB, et al. Pathogenesis of Escherichia coli diarrhea. N Eng J Med 1971; 285: 1.

30. Serény B. Experimental keratoconjunctivitis shigellosa. Acta Microbiol Acad Sci Hung 1957; 4: 367.

31. Kasmier L J.Estadística aplicada a la administración y la economía. McGraw Hill. México, 1978 